Check for updates

Cite this: J. Mater. Chem. C, 2020, 8, 328

Received 11th September 2019, Accepted 19th November 2019

DOI: $10.1039 / c 9 t c 05018 c$

rsc.li/materials-c

\title{
Dedoping-induced interfacial instability of poly(ethylene imine)s-treated PEDOT:PSS as a low-work-function electrode $\dagger$
}

\author{
Wanzhu Cai, (D) ab Thomas Österberg, ${ }^{b}$ Mohammad J. Jafari, (D) ${ }^{c}$ \\ Chiara Musumeci, (D) ${ }^{b}$ Chuanfei Wang, (D) ${ }^{d}$ Guangzheng Zuo, ${ }^{\text {e Xiaolong Yin, }}{ }^{a}$ \\ Xuhao Luo, ${ }^{a}$ Jim Johansson, ${ }^{b}$ Martijn Kemerink, (iD e Liangqi Ouyang, ${ }^{\text {b }}$ \\ Thomas Ederth $\left(\mathbb{D}^{c}\right.$ and Olle Inganäs ${ }^{b}$
}

\begin{abstract}
Transparent organic electrodes printed from high-conductivity PEDOT:PSS have become essential for upscaling all-carbon based, low-cost optoelectronic devices. In the printing process, low-work-function PEDOT:PSS electrodes (cathode) are achieved by coating an ultra-thin, non-conjugated polyelectrolyte that is rich in amine groups, such as poly(ethylene imine) (PEI) or its ethoxylated derivative (PEIE), onto PEDOT:PSS surfaces. Here, we mapped the physical and chemical processes that occur at the interface between thin PEIx (indicating either PEI or PEIE) and PEDOT:PSS during printing. We identify that there is a dedoping effect of PEDOT induced by the PEIx. Using infrared spectroscopy, we found that the amine-rich PEIx can form chemical bonds with the dopant, PSS. At lower PSS concentration, PEIx also shows an electron-transfer effect to the charged PEDOT chain. These interface reactions lock the surface morphology of PEDOT:PSS, preventing the redistribution of PSS, and reduce the work function. Subsequent exposure to oxygen during the device fabrication process, on the other hand, can result in
redoping of the low-work-function PEDOT:PSS interface, causing problems for printing reproducible Subsequent exposure to oxygen during the device fabrication process, on the other hand, can result in
redoping of the low-work-function PEDOT:PSS interface, causing problems for printing reproducible devices under ambient conditions.
\end{abstract} covices under ambient conditions.

\section{Introduction}

Low work-function (WF) electrodes processed from solutions are key components for various flexible organic semiconductor devices such as large-area organic solar cells. ${ }^{1}$ Different materials systems have been developed for this purpose. ${ }^{2}$ Many of the electrodes and devices are, however, fabricated at the lab-scale and under inert gas protection. ${ }^{2-4}$ Upscaling the appealing performance of these electrodes from lab-scale fabrication to largescale, industry-relevant production, however, is still challenging.

\footnotetext{
${ }^{a}$ Guangdong Provincial Key Laboratory of Optical Fiber Sensing and Communications, Guangzhou Key Laboratory of Vacuum Coating Technologies and New Energy Materials, Siyuan Laboratory, Department of Physics, Jinan University, Guangzhou 510632, P. R. China

${ }^{b}$ Biomolecular and Organic Electronics, Department of Physics, Chemistry and Biology, Linköping University, SE-581 83 Linköping, Sweden.

E-mail: liangqi.ouyang@liu.se, liangqi@kth.se

${ }^{c}$ Molecular Physics, Department of Physics, Chemistry and Biology, Linköping University, SE-581 83 Linköping, Sweden

${ }^{d}$ Surface Physics and Chemistry, Department of Physics, Chemistry and Biology, Linköping University, SE-58183 Linköping, Sweden

${ }^{e}$ Complex Materials and Devices, Department of Physics, Chemistry and Biology, Linköping University, SE-581 83 Linköping, Sweden

$\dagger$ Electronic supplementary information (ESI) available. See DOI: 10.1039/c9tc05018c
}

Problems associated with large area printed electrodes, including the overall low conductivity, instability in the long-term, poor charge selectivity and current leakage, all contribute to the performance loss in printed modules. ${ }^{5}$ To understand the mechanism leading to the loss of performance in the upscaling process is therefore a practical application-driven scientific investigation that benefits both processing and materials development.

One of the most widely used materials for printed semitransparent electrodes is poly(3,4-ethylene dioxythiophene):poly(styrene sulfonic acid) (PEDOT:PSS). With a record conductivity exceeding $4600 \mathrm{~S} \mathrm{~cm}^{-16}$ and superior stability, PEDOT:PSS has proven to be a promising material to replace the expensive indium tin oxide (ITO). ${ }^{7}$ There are very few competitive alternatives in terms of materials cost, processing conditions and mechanical compatibility. PEDOT:PSS is a polyelectrolyte complex consisting of positively charged PEDOT and negatively charged PSS. ${ }^{8,9}$ In this composition, PSS is in excess to PEDOT in order to stabilize the dispersion in aqueous solution. ${ }^{10}$ PSS is also essential for providing good film forming properties, for stabilizing the doping of PEDOT that is needed to achieve good conductivity and for acting as a dipole layer outside the PEDOT grain to modify the electrode work function. ${ }^{7}$ Currently, many types of PEDOT:PSS 
are commercially available, such as "Clevios P VP Al 4083" (4083) and "Clevios PH 1000" (PH1000). The PEDOT to PSS ratio of the former is $1: 6$, while the ratio of the latter is $1: 2.5$, according to the manufacturer instructions. The work function of a high-conductivity PEDOT:PSS electrode is $\sim 5.0 \mathrm{eV}$, which makes it suitable as an anode material for hole transport in many optoelectronic systems. To be used as an electron collecting electrode (cathode), however, the work function of PEDOT: PSS needs to be modified. ${ }^{11-14}$ Highly conductive PEDOT:PSS top-coated with a thin layer of non-conjugated polymers rich in amine groups, namely poly(ethylene imine) and derivatives (PEIx), is an easily processed but effective material to realize a cathode. ${ }^{4,15}$ However, the PEDOT:PSS based electrode was found to strongly influence the device stability. ${ }^{16,17}$ It is very important to address the problems and to understand the chemical and physical processes taking place at the interface.

In this work, we used both PEI and its ethoxylated derivative, PEIE (Fig. 1(d)), for the interfacial modification of highly conductive PEDOT:PSS electrodes in printed large-area organic solar cells. Both of them have been shown to be effective modification materials for creating low WF surfaces. ${ }^{4}$ When not specified, PEI and PEIE are collectively referred to as "PEIx". We directly printed PEIx solutions in polar solvents onto PEDOT:PSS electrodes as a thin modification layer to create the cathode for the assembly of solar cells. As we found that PEI generally gives better performance to the printed devices, we here focus on the effects of PEI on the morphology of PEDOT:PSS during consecutive printing. PEIE is also included in order to gain a complete picture of the mechanism of the change of the

(a)

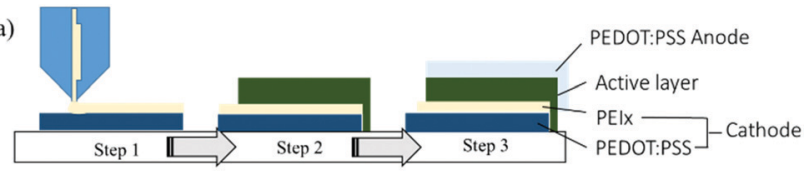

(b)

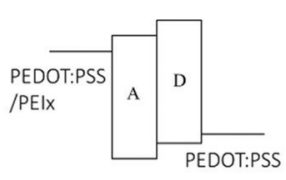

(d)

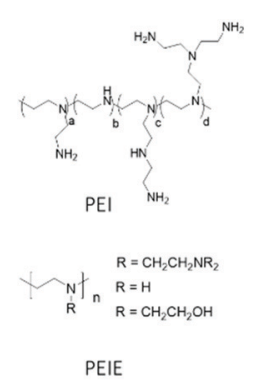

(e)

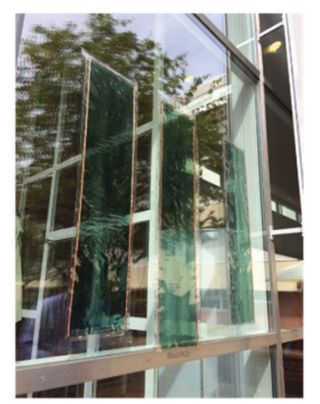

Fig. 1 (a) Schematic of PEI layer coated on PEDOT:PSS layer with slot die coating and the device printing process (3 steps). (b) Energy diagram of printed solar cells, A: electron acceptor, D: electron donor. (c) Chemical structures of PEDOT:PSS, PEDOT:tos and (d) PEI and PEIE. (e) Printed large area modules using the developed device structure and fabrication method reported in this work, photographed by the author. work function. We identified the mode of interactions between PEIx and PSS or PEDOT. We focused especially on the conditions for reproducibly upscaling high-performance devices.

\section{Results and discussion}

\subsection{Upscaling all-organic solar cells with slot-die coating}

The schematic of our printing process is shown in Fig. 1(a). Large-scale production of devices is achieved through layer-bylayer deposition of materials using slot-die coating on a flexible polyethylene terephthalate (PET) substrate. We use a device configuration of PEDOT:PSS/PEIx/active layer/PEDOT:PSS. Detailed descriptions of the materials and methods can be found in the Experimental Section. The devices were assembled through lamination, as previously reported. ${ }^{18}$ We fabricate the low-work-function electrode by printing an ultra-thin layer of PEI or PEIE from isopropanol (IPA) solutions directly onto PEDOT:PSS (Fig. 1(a)). It was proposed that this amine-rich thin layer creates a dipole layer at the electrode surface, resulting in a reduction of the $\mathrm{WF}^{4}$ Consequently, the electrode forms an Ohmic contact with the electron acceptor material (Fig. 1(b)). Although PEI and PEIE are both used in the literature for cathode modifications, we note that the PEI coating generally provides better device performance in our upscaling printing process. Compared to PEI, the ethoxylated derivative, PEIE, has a much decreased density of primary amine groups. It can nevertheless effectively reduce the WF of PEDOT electrodes to a similar value as that by PEI modification. As shall be discussed in the next sections, PEIE also leads to the dedoping of PEDOT. We therefore mainly focus on using PEI as the modification material for our printing process while this study included both PEI and PEIE. We assembled the full device by laminating the active materials coated on the anode side and the cathode side. This simple lamination technique allows us to assemble large area functional devices. ${ }^{18}$ Fig. 1(c and d) shows the related molecular structures of the materials used in this work. Fig. 1(e) shows a picture of the final OPV module.

\subsection{The modes of interactions between PEDOT:PSS and PEIx}

We use polarization-modulation infrared reflection-absorption spectroscopy (PM-IRRAS) to trace the interfacial interactions between the PEDOT:PSS electrodes and PEIx (Fig. S2, ESI $\dagger$ ). We selected three types of PEDOT:PSS electrodes that are relevant to organic electronic devices: Clevios PH4083, PH1000, and PH1000 films rinsed with isopropyl alcohol (IPA) (referred to as PH1000R in the following text). The PEDOT:PSS aqueous dispersion is described as a microgel system where the conducting PEDOT core is stabilized by an insulating PSS shell. ${ }^{8}$ The electronic properties of the PEDOT:PSS films are strongly affected by the phase separation between these two components. Previously, we have shown that a portion of free PSS is separated from PEDOT:PSS-rich domains when the material is processed in an aqueous dispersion with additives (diethylene glycol, ethylene glycol or dimethyl sulfoxide). ${ }^{19}$ This phase separation is critical to achieve high conductivity films. ${ }^{20}$ Selective removal of PSS from 
(a)

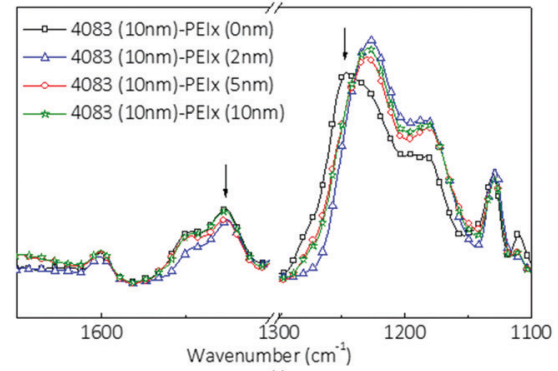

(b)

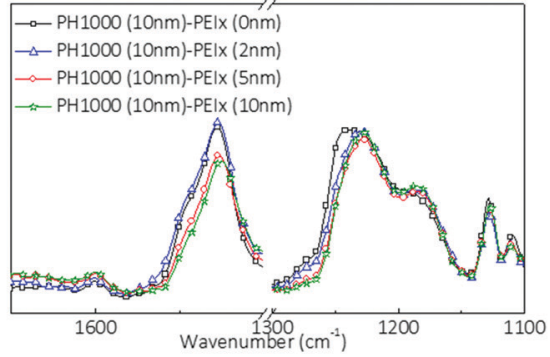

(c)

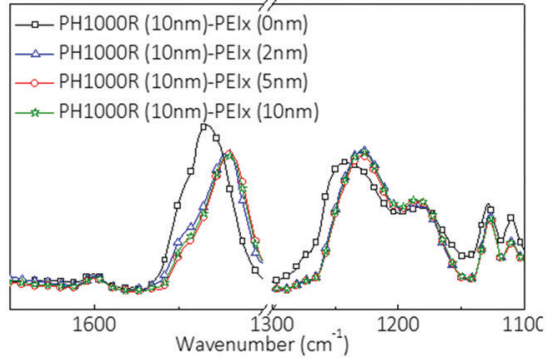

Fig. 2 PM-IRRAS spectra of (a) PH4083, (b) PH1000 and (c) rinsed PH 1000 (PH1000R) with PEIE coatings of different thicknesses.

PEDOT:PSS has also been reported. ${ }^{21}$ We select PH4083 in our study as it is a widely used hole-transport layer material, and importantly, compared to PH1000, it has a PSS-to-PEDOT weight ratio that increased from $2.5: 1$ to $6: 1$, which will result in a considerably increased portion of free PSS in the corresponding film. On the other hand, we use IPA rinsing to further decrease the PSS concentration on the PEDOT:PSS (PH1000) surface (Fig. S1 and Discussion, ESI $\dagger$ ). We coated PEIx onto these electrodes at different PEIx thicknesses. As shown in Fig. 2(a-c), the coating causes shifts of the IR absorption bands around 1530 and $1245 \mathrm{~cm}^{-1}$ (indicated with arrows in Fig. 2(a)). These two bands are related to the asymmetric $\mathrm{C}=\mathrm{C}$ vibration of the thiophene ring in PEDOT, and the $\mathrm{SO}_{3}$ stretching in PSS, respectively. For all uncoated films, there is also an influence of PSS concentration on the intensity and positions of the $1530 \mathrm{~cm}^{-1}$ band (PEDOT) absorptions. After PEIx coating, this band shifted to lower frequency with decreased intensities. This change is related to the switching of the PEDOT from a doped quinoid to neutral benzenoid structure, suggesting that a portion of PEDOT in the thin films is dedoped by the PEIx coating. ${ }^{22,23}$ We found that the degree of dedoping, as revealed by the change in band position, is inversely correlated to the PSS concentration. Meanwhile, the shift in the $\mathrm{SO}_{3}$ bond from 1245 to $1227 \mathrm{~cm}^{-1}$ indicates that the $\mathrm{SO}_{3}$ groups in PSS react with the amines in PEIx to form new bonds. Previously, Fabiano et al. found that commercial PEI contains volatile small-molecule impurities (identified as ethyleneimine dimers and trimers) that can also dedope PEDOT. This was demonstrated by exposing PEDOT films to heated PEI vapor. ${ }^{22} \mathrm{We}$ have observed here dedoping effects of solution processed, ethoxylated PEI (PEIE) on PEDOT, which we attributed to the interactions between the amine groups of the polymer coating and the PSS.

To elucidate the respective contributions of PEDOT dedoping and PEIx:PSS complex formation at the PEIx:(PEDOT:PSS) interface, we performed an interfacial interaction principal component analysis (PCA) on the data sets obtained on these thin films. This was carried out in two wavenumber regions, corresponding to the thiophene ring vibrations (1600 to $\left.1450 \mathrm{~cm}^{-1}\right)$ and the $\mathrm{SO}_{3}(\mathrm{~S}=\mathrm{O})$ stretching in PSS (1300 to $\left.1100 \mathrm{~cm}^{-1}\right)$. Two-dimensional score plots from PCA modeling are shown in the ESI $\dagger$ (Fig. S2). The location of the points (each point corresponding to a specific sample) in the score plots represents the variability (statistical variation) of each spectrum with respect to the mean of the spectra. Thus, the position of a point reflects some characteristic of the corresponding sample, and grouping of points indicates the existence of samples with similar characteristics over the considered spectral region. ${ }^{24,25}$ The first principal component (PC1), which expresses most of the variance in the raw IR data, allows discrimination between the different doping states of PEDOT:PSS, as identified from the grouping of the corresponding data points along the PC1 axis, explaining $62 \%$ of the variation in both of the considered spectral regions. The second principal component (PC2) is due to the interfacial interactions between PEDOT or PSS and PEIx, explaining $27 \%$ and $21 \%$ of the variation in the $1300-1100 \mathrm{~cm}^{-1}$ and $1600-1450 \mathrm{~cm}^{-1}$ spectral regions, respectively. To understand the dependence of the interfacial interactions on PEIx thickness, the change in PC2 versus PEIx thickness is plotted in Fig. 3(a and b) for the two wavenumber regions. PC2 for the $\mathrm{SO}_{3}$ stretching in PSS (Fig. 3(a)) changes considerably with PEIx thickness up to $1.5 \mathrm{~nm}$, but it does not vary further for thicker PEIx coatings (Fig. 3(a)). This suggests that the interaction occurs within the first $2 \mathrm{~nm}$ from the interface. The concentration of PSS in the PEDOT:PSS layer also affects the PSS-PEIx interactions. We find the largest change in PC2 relating to $\mathrm{SO}_{3}$ stretching in $\mathrm{PH} 4083$, which has the highest PSS concentration. The PC2 obtained from analysis of the thiophene $\mathrm{C}=\mathrm{C}$ vibration region in PEDOT shows an opposite trend with different PEIx coating thicknesses (Fig. 3(b)). In both types of PH1000, either with or without rinsing, there is significant de-doping of PEDOT due to the PEIx coating, whereas in PH4083, no major change was found. These results show that the PEIx coating has two types of interactions with PEDOT:PSS. It transfers electrons to dedope PEDOT, and it forms bonds with PSS through the electron-rich amine groups. At high PSS concentration (in 4083), the reaction between PSS and PEIx is dominant over the PEDOT-PEIx interaction. Also, when the PSS concentration decreases, de-doping becomes more dominant.

The absorption spectra of PH1000 top-coated with 0.1 wt $\%$ PEI and $0.5 \mathrm{wt} \%$ PEI (both spin-coated at $2000 \mathrm{rpm}$ ) were compared with that of 4083. As shown in Fig. S3 (ESI $\dagger$ ), coated with $0.1 \%$ PEI, $\mathrm{PH} 1000$ film showed a large decrease of absorption close to $900 \mathrm{~nm}$, which suggests a decrease in polaron concentration. For PH4083 

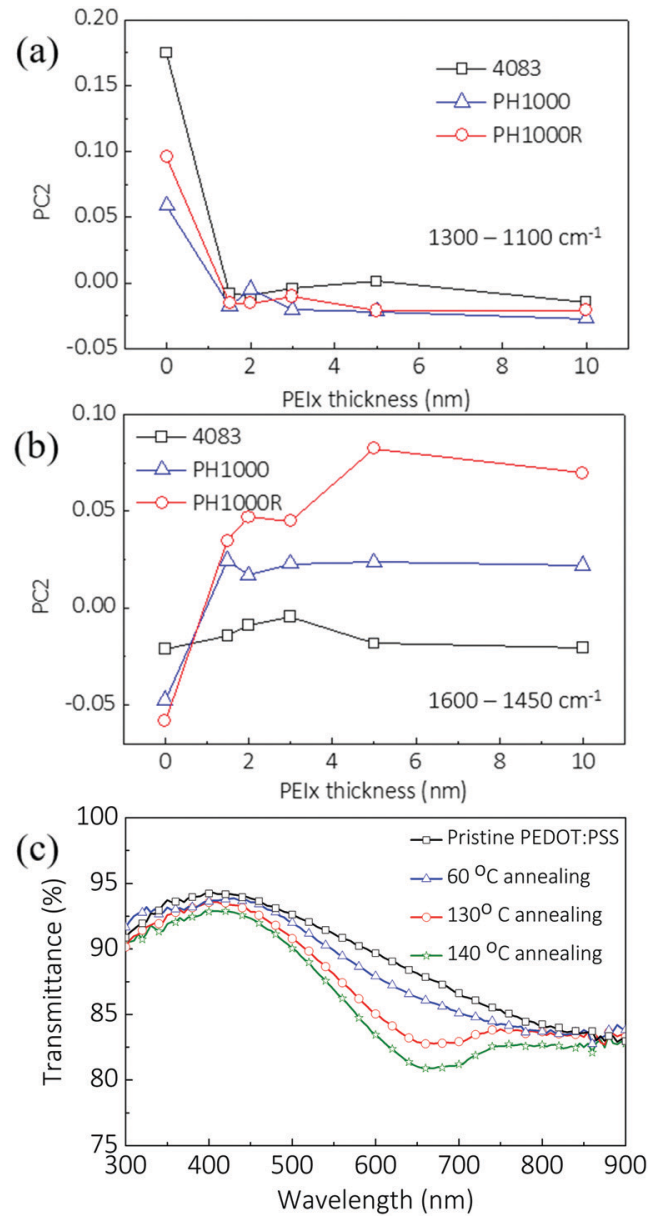

Fig. 3 (a) and (b) Principal component analysis (PCA) of FTIR spectra of the PEDOT films (see Fig. S2, ESI $\dagger$ ), showing the scores of the second component (PC2) versus PEIE thicknesses. Data were generated across the two fingerprint sub-regions: (a) $1300-1100 \mathrm{~cm}^{-1}$, with $\mathrm{PC} 1=61.9 \%$ and $\mathrm{PC} 2=27.2 \%$, and (b) $1600-1450 \mathrm{~cm}^{-1}$, with $\mathrm{PC} 1=62.7 \%$ and $\mathrm{PC} 2=21.1 \%$. (c) Optical transmission spectrum of PEDOT:PSS coated with PEI with different annealing temperatures. Thickness was measured by ellipsometry.

coated with $0.1 \%$ PEI solution, the absorption in this range was relatively unchanged. This is possibly a sign that the dedoping process is suppressed in 4083 . However, with PEI concentration increasing to $0.5 \%$, both films show clearly enhanced absorption at $600 \mathrm{~nm}$, corresponding to the $\mathrm{Pi}^{-\mathrm{Pi}^{*}}$ absorption of the thiophene ring in neutral PEDOT. This observation indicated that PEI can saturate PSS and continue to dedope PEDOT.

As a degenerate semiconductor, the conductivity of PEDOT: PSS decreases when it is dedoped. We found, however, that the $2 \mathrm{~nm}$ thick PEIx coating induced only negligible changes in the PEDOT conductivity. We surmise that this is because the dedoping is limited at the thin interface between the two layers. To understand the conductivity change and the de-doping process related to PEIx coating, we spin-coated PEIE from its 0.01-5 wt\% IPA solutions onto PH1000 films and evaporated gold contacts onto these films for measurements of Seebeck coefficient and the conductivity in an inert atmosphere.

Fig. S4 (ESI $\dagger$ ) shows the electrical conductivities and Seebeck coefficients of these films. We found that the conductivities and
Seebeck coefficients of PH1000 films stayed almost constant after 0.1 wt $\%$ PEI coating (which gives a PEIx coating of $\sim 2 \mathrm{~nm}$ thickness), whereas a decrease in conductivity was found for coatings with higher PEIE concentrations. When the PEIE coating was deposited from $5 \mathrm{wt} \%$ solution, the conductivity of PH1000 is around $7 \mathrm{~S} \mathrm{~cm}^{-1}$, which is almost 2 orders of magnitude lower than that of the pristine PH1000 film. Importantly, the Seebeck coefficient increased up to over $60 \mu \mathrm{V} \mathrm{K} \mathrm{K}^{-1}$ from $30 \mu \mathrm{V} \mathrm{K} \mathrm{K}^{-1}$ for pure PH1000.

Note that the conductivity $(\sigma)$ and Seebeck coefficient $(S)$ of organic semiconductors are closely related to the Fermi energy $\left(E_{\mathrm{F}}\right)$ and transport energy $\left(E_{\mathrm{tr}}\right)$ of the materials. A simple expression is given as follows: ${ }^{26}$

$$
\sigma \propto \exp \left(\frac{E_{\mathrm{F}}-E_{\mathrm{tr}}}{T}\right) \text { and } S \propto \frac{E_{\mathrm{F}}-E_{\mathrm{tr}}}{T} .
$$

Here, $T$ is the temperature and $E_{\mathrm{F}}$ is a function of charge density. Assuming that the transport energy stays constant, the conductivity and Seebeck coefficient are then dependent on the charge density in the devices. Therefore, as below $0.1 \mathrm{wt} \%$ PEIE concentration, the coating showed negligible effects on the conductivity of PEDOT films, the charge density can be described as constant. The simultaneous change of both conductivity and Seebeck coefficient at higher PEIE concentrations suggests a decrease in charge density of the doped semiconducting material. We therefore propose that the decrease in conductivity is related to a de-doping effect of PEIE on the PH1000 films. We also built inverted structures with 5 wt\% PEIE coatings at the bottom of PH1000 films. We expect that this geometry will remove the possible influences of the insulating nature of the PEIE coating on top of PEDOT. Fig. S3 (ESI $\dagger$ ) also shows the conductivities and Seebeck coefficients of the inverted bilayers. A significant decrease ( 2 orders of magnitude) in conductivity, compared to that of the pure PH1000 film also occurs in the inverted structures, which further confirms the de-doping effect of PEIE on PEDOT:PSS film.

Similar de-doping effects were confirmed for PEI coatings on thick solution processed PEDOT:PSS electrodes. Fig. 3(c) shows the optical transmission spectrum of a PH1000 layer (around $100 \mathrm{~nm}$ ) coated with a PEI layer. When the annealing temperature increases, which means the molecular diffusion or interaction will be more pronounced, ${ }^{26}$ a decrease in the transmission at around $600 \mathrm{~nm}$ is observed. This is consistent with the de-doping results, because the $\pi-\pi^{*}$ absorption of the thiophene ring in neutral PEDOT increases.

The conductivity of 4083 coated with PEI is presented in the ESI. $\dagger$ The resistances were obtained by fitting the $J-V$ curve of devices with Ohm's law. When the PEI coating was thin PEI, there was only a limited change in the conductivity. As the PEI coating became thicker, we found a decrease in conductivity. This result is consistent with the observation of absorption in Fig. S3 (ESI $\dagger)$.

\subsection{Influences and implications of oxidation states on the work function of PEDOT electrodes}

It has been reported that PEI induces a color shift and a decrease in conductivity of PEDOT:PSS films. ${ }^{26,27}$ We have 
provided here spectral evidence of dedoping effects of the PEIx on PEDOT:PSS electrodes. The oxidization state (doping level) of a conjugated polymer has a positive correlation to its work function. ${ }^{28-31}$ To elucidate the contributions of oxidation states to work function and to minimize the influence of excess PSS dopant, we included the PEDOT:tosylate (PEDOT:tos) film in this work as another model example. Fig. 1(c) shows the composition of PEDOT:tos. Compared to PEDOT:PSS, PEDOT:tos is free of the dipole-forming PSS on its surface ${ }^{7}$ and has a much decreased dopant concentration (the molar ratio of PEDOT to tosylate is estimated to be around 1:0.3) in the polymer matrix. We chemically removed the dopant from PEDOT:tos with hydrazine rinsing and observed the suppression of polaron and bipolaron bands. Meanwhile the dedoped PEDOT shows a new peak around $600 \mathrm{~nm}$. Importantly, de-doping of the bulk film of PEDOT:tos decreased the work function by around $0.45 \mathrm{eV}$ (Fig. 4(c) and Fig. S5, ESI $\dagger$ ).

Oxygen in the air has been shown to effectively redope PEDOT:tos. ${ }^{23,32,33}$ As a result, the WF of dedoped PEDOT:tos quickly increases in the presence of oxygen, as also observed by other groups. $^{23,32}$ We monitored the WF change of PEIx modified PEDOT:tos with different doping levels under ambient conditions. We note that PEIx coating on PEDOT:tos causes much smaller changes in WF. $10 \mathrm{~nm}$ of PEIx coating decreased the WF of pristine (oxidized) PEDOT:tos by $0.25 \mathrm{eV}$ (from $\sim 4.8$ to $\sim 4.6 \mathrm{eV}$ ). We attribute the smaller decrease in WF to the quick redoping of oxygen at the PEIx:PEDOT:tos interface. On the other hand, a PEIx coating on freshly prepared dedoped PEDOT:tos slightly decreased the WF by $0.1 \mathrm{eV}$. The change of WF of coated PEDOT:tos in air was monitored over a period of 3 to 5 hours. As shown in Fig. 4(c) and (d), with the PEIx coating, the WF of dedoped PEDOT:tos increased by $0.2 \mathrm{eV}$ in the first 20 minutes and continued to increase under ambient conditions. This suggests that the bulk redoping of PEDOT has a significant impact on the WF at the interface. Although the WF of PEI modified pristine PEDOT:tos was relatively stable at $4.6 \mathrm{eV}$, this small decrease in $\mathrm{WF}$ compared to unmodified PEDOT.tos, however, could be because the redoping of the thin PEDOT:PEI interface may have immediately taken place in the air.

The results of PEDOT:tos suggest that dedoping plays an important role in the low WF electrodes and even with PEIx coating on the surface, redoping can lead to an increase in WF. As discussed, it was well documented that PEIx coating on PEDOT:PSS films induces small but significant changes in film absorption, which is characteristic of dedoped PEDOT films. ${ }^{22,26}$ To relate these observations to the actual device performance, we first coated PEIx onto PH1000 films that were rinsed with IPA and compared them with pristine PH1000 films. As will be discussed in the next section, IPA rinsing removes excess free PSS from PH1000 and induces a moderate decrease of the WF by $0.1 \mathrm{eV}$ (Fig. S1, ESI $\dagger$ ). After PEIx coating, the shift in WF was very similar for both films: it decreased by $0.49 \mathrm{eV}$ and $0.50 \mathrm{eV}$, respectively (Fig. S1, ESI $\dagger$ ).

We then monitored the WF change of PH1000 induced by PEIx coating at different thicknesses. 2 nm of PEIx on PH1000 decreased the WF by $0.5 \mathrm{eV}$ (Fig. 4(b)). Increasing PEIx (a)
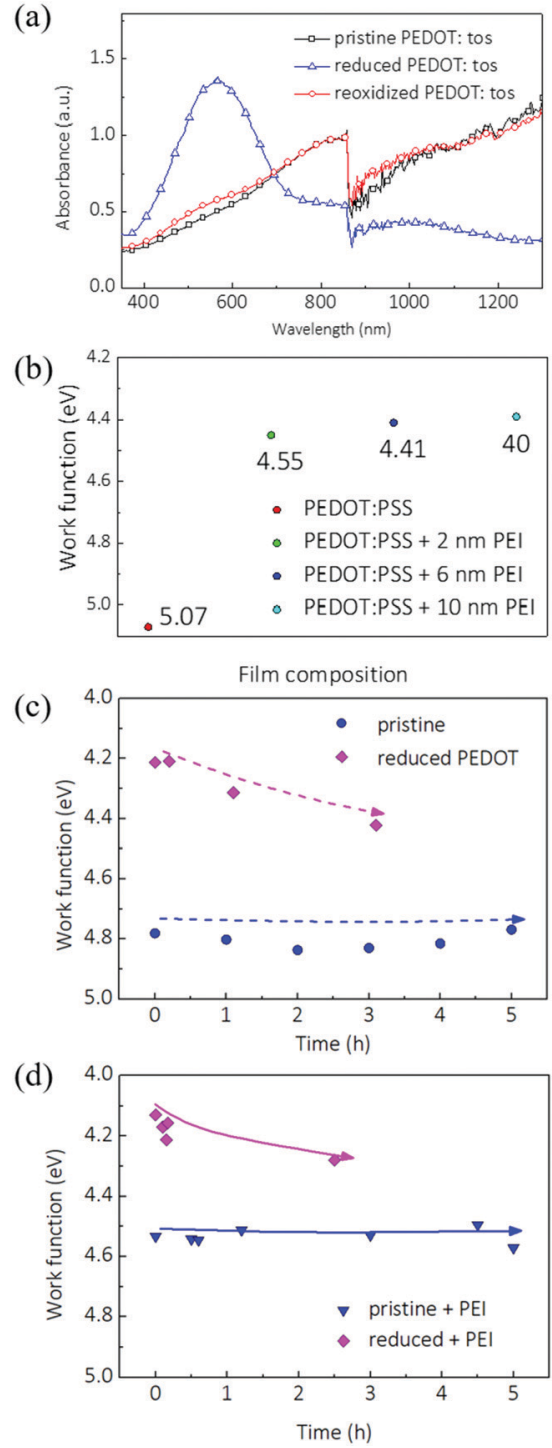

Fig. 4 (a) UV-vis-NIR spectra of pristine, half reduced and fully reduced PEDOT:tos. (b) WF of PEDOT:PSS films with different thicknesses of PEI layer. (c) WF of pristine and reduced PEDOT:tos films over time. (d) WF of PEI coated PEDOT:tos films over time.

thickness to $10 \mathrm{~nm}$ caused a further $0.15 \mathrm{eV}$ decrease. There are only minimal effects upon even thicker PEIx coating, which suggests that the decrease in WF is mainly contributed by the first $2 \mathrm{~nm}$ at the PEIx:PEDOT:PSS interface. The color shifts and WF changes are consistent with those of dedoped PEDOT.

We then constructed representative OPV devices with PEIx modified PEDOT:PSS electrodes, using a lamination technique. ${ }^{15}$ We carried out all the processes under ambient conditions at room temperature. The PEDOT:PSS electrodes were first coated with a PEIx layer. After drying in a glove box, they were taken out and exposed to ambient conditions for a time ranging from 0 to $30 \mathrm{~min}$, before being assembled into an OPV device. The device performances are summarized in Table 1, and the corresponding $J V$ characteristics are plotted in Fig. S1(c) (ESI $\dagger$ ). Clearly, when the low WF electrodes were exposed to air, $V_{\text {OC }}$ of the device 
Table 1 Performance parameters of devices based on PEDOT:PSS/PEI electrode; exposure time refers to the duration time before the active layer deposition. All processes were carried out in air. The performance parameters are average values based on five cells

\begin{tabular}{lllll}
\hline Exposure time & $V_{\mathrm{OC}}(\mathrm{V})$ & $J_{\mathrm{SC}}\left(\mathrm{mA} \mathrm{cm}^{-2}\right)$ & PCE $(\%)$ & $\mathrm{FF}$ \\
\hline Reference & 0.74 & 7.40 & 1.70 & 0.31 \\
$10 \mathrm{~min}$ & 0.72 & 4.28 & 0.83 & 0.27 \\
$20 \mathrm{~min}$ & 0.72 & 6.73 & 1.37 & 0.28 \\
$30 \mathrm{~min}$ & 0.65 & 5.59 & 0.98 & 0.27
\end{tabular}

decreased from $0.74 \mathrm{~V}$ to $0.65 \mathrm{~V}$. The overall power conversion efficiency (PCE) also dropped from $1.70 \%$ to $0.90 \%$. To reduce the influence of humidity, the PEIx modified electrodes were also dried at $60{ }^{\circ} \mathrm{C}$ for a time ranging from 0 to 30 minutes. Elevated temperatures will accelerate the oxygen redoping of the modified electrodes. Heating at $60{ }^{\circ} \mathrm{C}$ caused a larger change of the device performance (Table S1, ESI $\dagger$ ). After 30 minutes of heating, the $V_{\text {OC }}$ dropped from $0.74 \mathrm{~V}$ to $0.62 \mathrm{~V}$. The fill factor dropped from 0.31 to 0.26 . The overall efficiency dropped from $1.70 \%$ to $0.80 \%$ with the decreasing $J_{\mathrm{SC}}$ and FF. We note that as the devices were constructed through lamination, the area is not strictly defined. The performances of these modules can also be improved by optimizing the processing conditions. ${ }^{34}$ We then repeated the experiment with evaporated top electrodes as well as another active materials system that has a defined area and obtained similar results (Table S2, ESI $\dagger$ ).

\subsection{PEIx pinning of PEDOT:PSS morphology}

As discussed in Section 2.2, the PEDOT:PSS film consists of two components: reorganized PEDOT:PSS and free PSS. We found that in the dry film, a portion of free PSS can also be extracted from the PEDOT:PSS matrix and segregate to the surface during the subsequent solution printing. Fig. 5(a-d) show an example
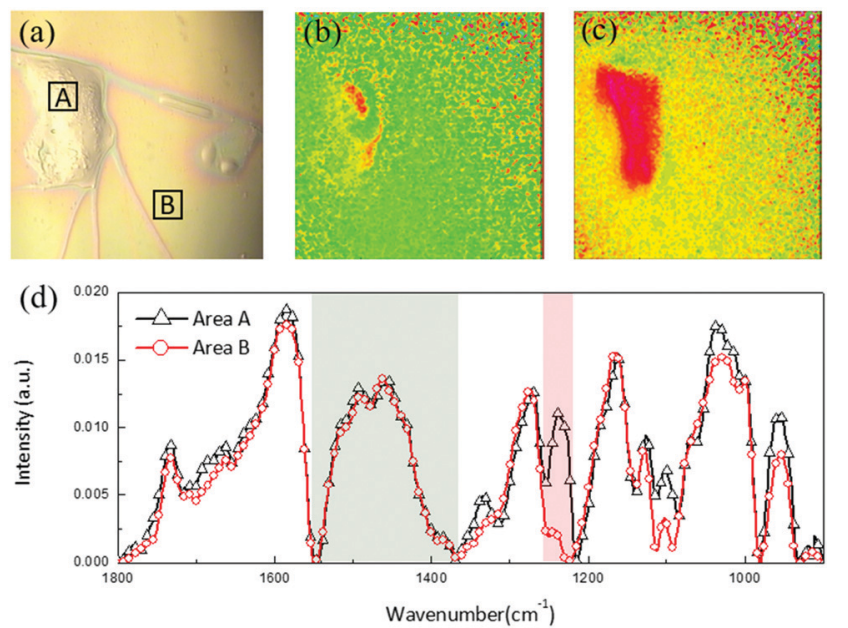

Fig. 5 Polar solvent removes and redistributes free PSS in highly conductive PEDOT:PSS electrodes. (a) Reflection mode optical microscopy. Relative absorption mapping of $(b)$ the $\mathrm{C}=\mathrm{C}$ stretching from PEDOT (integrated from 1500-1400 $\mathrm{cm}^{-1}$ ) and (c) the $-\mathrm{S}=\mathrm{O}$ stretching in PSS (integrated from $1282-983 \mathrm{~cm}^{-1}$ ) from a PEDOT:PSS film where water has been deposited and left to evaporate. (d) IR spectra of areas A and B in (a). when a water droplet is cast on the PEDOT:PSS electrode surface. The dried water droplet induced accumulation of a large material cluster $(\sim 100 \mu \mathrm{m})$ on the film surface. We imaged the chemical composition of the cluster with infrared (IR) microspectroscopy. As shown in Fig. 5(d), a comparison of IR spectra taken from the cluster (area A in Fig. 5(a)) and the local background (area B) reveals that both regions show a characteristic PEDOT thiophene ring $\mathrm{C}=\mathrm{C}$ stretching around 1500 to $1400 \mathrm{~cm}^{-1}$ (indicated by the gray box). The absorption band around $1240 \mathrm{~cm}^{-1}$ (pink box) is attributed to the $\mathrm{S}=\mathrm{O}$ stretching from the $\mathrm{SO}_{3}$ group in free PSS. Compared to the PCA analysis, here, we integrate over wider wavenumber ranges for PEDOT and PSS, respectively, to better account for the concentration variations of both components in the film. Fig. 5(b and c) show the images of integrated IR absorptions from the two characteristic bands over the whole area. These results indicate that while PEDOT is uniformly distributed in the film and unaffected by the solvent, the cluster is mainly accumulated "free" PSS, confirming our observation that free PSS can be extracted during consecutive solution printing.

To understand the influences of free PSS on the subsequent printing of the PEIx layer, we first printed a PEDOT:PSS film on a plastic substrate coated with a reflective gold layer for IR imaging. The PEIx modification layer was then printed onto the PEDOT:PSS electrodes from isopropanol solution. We also printed pure isopropanol onto PEDOT:PSS under the same processing conditions as a control. The continuous PEDOT:PSS film was not damaged during the IPA coating process. No defects, cracks or pinholes were found on these films. However, the printing process leads to several distinct regions: the edge of pure IPA or PEIx, the coated PEDOT:PSS, the edge of coated PEDOT:PSS and unaffected regions of PEDOT:PSS. Fig. 6(a and b) show optical images of the films after IPA coating and PEIx coating, respectively. We mapped the IR spectra of these samples to understand the distribution of materials across the printed stripe. We used PCA to reduce the dimensionality of the data and to simplify the interpretation of the IR spectra. PCA was performed in the wavenumber region of $4000-900 \mathrm{~cm}^{-1}$. Fig. 6(c and d) show the three principal components versus the relative position across the film, where PC1 as the most important score in explaining the variability in the data set represents the effect of thickness as the most influential physical variable on overall spectral intensity. PC2, representing the second largest source of variance in the data, expresses typical thiophene ring vibrations (the highest structural density in the samples), which is evidence of the existence of PEDOT. Finally, PC3 is associated with $\mathrm{SO}_{3}$ and other ring vibrations in PSS, which reflects the density of PSS in the samples.

Comparing Fig. 6(c) and (d), the IPA solvent coating has a limited effect on the thickness while PEIx coating caused a visible increase in film thickness, clearly due to the PEIx deposition on PEDOT:PSS. Similarly, the IPA rinsing did not cause any change in PC2, reflecting the PEDOT content of PEDOT:PSS. However, an increase of PC2 (PEDOT contribution) is seen when going from the border of the PEIx coating to the bulk of the PEIx modified film in Fig. 6(d). As expected, 
(a) IPA

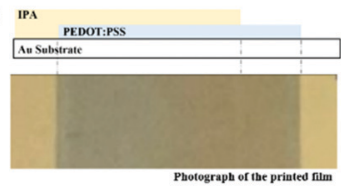

(c)

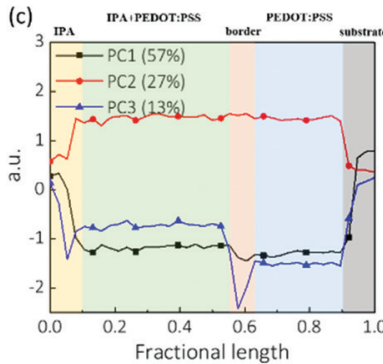

(b)
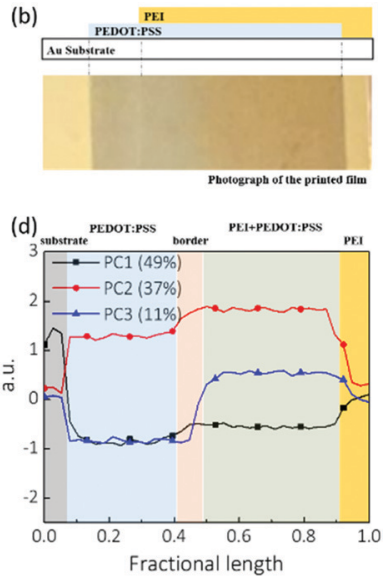

Fig. 6 (a) Photograph of a printed PEDOT:PSS strip with a solvent (IPA) strip printed on top. (b) Photograph of a printed PEDOT:PSS strip with a PEI strip printed on top. (c) The first three components (PC1, PC2 and PC3) versus the position, corresponding to the sample with the IPA strip on PEDOT:PSS as shown in (a). PC1 $=57 \%$, PC2 $=27 \%$, and PC3 $=13 \%$. (d) The first three components (PC1, PC2 and PC3) versus the position, corresponding to the sample with the PEI strip on PEDOT:PSS as shown in (b). $\mathrm{PC1}=44 \%, \mathrm{PC} 2=32 \%$, and PC3 $=24 \%$. The samples were printed on gold coated reflective substrates.

PC3 analysis (Fig. 6(c)) shows that after IPA solvent printing, PSS has accumulated at the borders of the film, reminiscent of the "coffee ring" effect.

Extraction and accumulation of PSS on the film may increase the film roughness, leading to non-uniform deposition of sequential layers. $^{35}$ As a too thick insulating PSS layer can block carrier diffusion, it can also result in an S-shaped $J V$ curve. ${ }^{36}$ Interestingly, when a PEIx in IPA solution was coated, the accumulation of PSS disappeared. PC3 analysis (Fig. 6(d)) on PEIx coated PEDOT:PSS films shows that the PSS component changes uniformly across the whole PEIx covered area. We attribute this morphology difference to the interaction between the PSS components in PH1000 and the coated PEIx, because their chemical bonding can suppress the migration and accumulation of extracted PSS.

\section{Experimental}

\subsection{Film and device fabrication}

All the films were deposited by slot die coating in air, as shown in the schematic in Fig. 1(a). A mini-slot die coater developed by FOM TECHNOLOGIES was employed for this work. The running speed of the web was $1 \mathrm{~m} \mathrm{~min}^{-1}$ with no tension and substrate heating applied. A syringe pump was used to inject the solution into the slot die head. The opening of the slot die coater is $13 \mathrm{~mm}$ and the pumping rate is $0.8 \mathrm{ml} \mathrm{min}^{-1}$. The high conductive grade PEDOT:PSS (PH 1000) was purchased from Heraeus, in which a weight ratio of PSS to PEDOT was $1: 2.5$. 6 vol\% polyethylene glycol and 5 vol\% silquest A186 silane were mixed into the PEDOT:PSS solution. The film thickness was $\sim 200 \mathrm{~nm}$ with a transmission of $90 \%$ and conductivity of $300 \mathrm{~S} \mathrm{~cm}^{-1}$. PH 1000 was used both as the anode and the cathode. PEIx stock solutions were purchased from Sigma-Aldrich.

The solutions were diluted with isopropanol (IPA, Sigma-Aldrich) to a concentration of $1 \mathrm{mg} \mathrm{ml} \mathrm{m}^{-1}$. For IPA and PEIx printing on PEDOT:PSS, the ink pumping rate and substrate speed are the same. When not specified, the films were annealed at $80{ }^{\circ} \mathrm{C}$. The thickness of PEIx was measured by ellipsometry. PEDOT:PSS with PEIx modification was used as the cathode, and the pristine PEDOT:PSS films were used as the anode side. The active layer material was (poly[[2,3-bis(3-octyloxyphenyl)-5,8-quinoxalinediyl]2,5-thiophenediyl]) (TQ1): PC61BM (weight ratio of 1:1), which was dissolved in $O$-xylene with a mass concentration of $20 \mathrm{mg} \mathrm{ml}^{-1}$. Molecular structures of TQ1 and $\mathrm{PC}_{61} \mathrm{BM}$ are shown in Fig. S7 (ESI $\dagger$ ). We constructed the solar cell structure of PET/ PEDOT:PSS/PEIx/TQ1:PC61BM/PEDOT:PSS using a lamination technique we previously developed. ${ }^{15}$ Both top and bottom PEDOT: PSS electrodes are PH1000.

\subsection{Device measurement}

$J V$ curves were measured using a Keithley 2400 source meter with the solar cell illuminated under simulated AM1.5 solar illumination $\left(100 \mathrm{~mW} \mathrm{~cm}^{-2}\right)$.

\subsection{Fourier-transform infrared (FTIR) measurements}

IR microspectroscopy was performed using a Bruker Hyperion 3000 microscope in reflection mode, using a Bruker Tensor 27 as a light source. The microscope has a 128 by 128 pixel focal plane array detector, and the whole system is continuously purged with nitrogen. IR images were captured with a $15 \times$ objective, resulting in a $300 \times 300 \mu \mathrm{m}^{2}$ field of view, imaging $2.3 \times 2.3 \mu \mathrm{m}^{2}$ per pixel. Spectra were acquired at $4 \mathrm{~cm}^{-1}$ spectral resolution between 4000 and $1000 \mathrm{~cm}^{-1}$ and by summing 64 scans. Background images were obtained on the bare goldcoated portion of the substrate film. A rubber-band baseline correction was used for all data, and data analysis was performed using the Bruker OPUS software (v. 7.2). Polarization-modulation infrared reflection-absorption spectroscopy (PM-IRRAS) measurements were performed at an angle of incidence of $86^{\circ}$ from the surface normal with a variable-angle reflection accessory in a Bruker PMA50 system with a liquid nitrogen-cooled MCT detector connected to a Bruker Vertex 70 spectrometer. Incident light was modulated with a photoelastic modulator (Hinds: ZnSe $50 \mathrm{kHz}$ ), and acquired signals were demodulated using a lock-in amplifier (Stanford Research SR830). Data were collected between 4000 and $800 \mathrm{~cm}^{-1}$, and baseline-corrected by identical rubberband corrections over the spectral range.

Principal component analysis (PCA) was used to reduce the dimensions of data into a series of orthogonal eigenvectors that are linear functions of the original variables, and which represent the directions of the greatest variances in the data, while retaining the information about the variations in data points. ${ }^{24}$ PCA enables identification and interpretation of FTIR spectra of samples by reducing hundreds of absorbance values at corresponding spectral wavenumbers into a single point. PCA was carried out for identification of spectral variations in the different wavenumber regions. Three principal components (PCs) were chosen for data analysis, and each PC describes the spectral variability among the samples, in decreasing order. After baseline correction, data were normalized 
to reduce the instrumental and environmental effects (using Bruker OPUS 7.2 software). Spectral data reduction via principal components analysis (PCA), and model-based clustering were performed using linear discriminant analysis (LDA) for multigroup classification; then, PCA was employed to compress the large number of correlated wavenumbers into a new reduced data set. This procedure was applied for different spectral regions to study the different interfacial interactions.

\subsection{UPS measurement}

UPS measurement was carried out in a dedicated home designed and built spectrometer with a setting that yields a width of $0.08 \mathrm{eV}$ of the Fermi edge of polycrystalline gold recorded at room temperature. In UPS measurement, the source of photons used is typically $\mathrm{HeI}(h \nu=21.2 \mathrm{eV})$ and the work function of the film was extracted from the edge of the secondary electron cutoff of the UPS spectrum by applying a bias of $-49.9 \mathrm{~V}$ to the samples.

\subsection{AFM and Kelvin probe measurement (KPM)}

KPFM was performed using a Dimension 3100 scanning probe microscope equipped with a NanoScope IV controller. Measurements were performed in amplitude modulation, using the "two-pass" interleave mode. Pt-coated silicon probes having a nominal spring constant of $3 \mathrm{~N} \mathrm{~m}^{-1}$ and resonance frequency of $75 \mathrm{kHz}$ were used. Freshly cleaved highly oriented pyrolytic graphite (HOPG) was used for calibration. All measurements were performed in air, at room temperature. In order to minimize the influences of moisture, and the surface potential measurements were taken immediately after the heating of the samples. In Fig. S7 (ESI $\dagger$ ), every data point is the average of 100 readings of the surface potential. The influence of the moisture cannot be fully avoided.

\subsection{Conductivity and Seebeck coefficient measurement}

All the films were spin-coated under ambient conditions, and PEIx layers were deposited on the top of pre-casted PH1000 films from IPA solutions with different concentrations. The inverted devices were made by first spin-coating a PEIx layer onto the substrate followed by the deposition of the PH1000 film. After that, all the films were thermally annealed at $130{ }^{\circ} \mathrm{C}$ for 30 minutes. $\mathrm{Au}(80 \mathrm{~nm})$ electrodes were thermally evaporated onto the films through a shadow mask under a pressure of $1 \times 10^{-6} \mathrm{mbar}$. The contact dimensions are $2 \times 7 \mathrm{~mm}$ (width $\times$ length) with a gap of $0.5 \mathrm{~mm}$ between two neighboring contacts; they therefore define channel dimensions of $7 \times$ $0.5 \mathrm{~mm}$. Room temperature electrical characterizations were performed in a glove box under a $\mathrm{N}_{2}$ atmosphere. The methodology used for the Seebeck coefficient measurement is the same as that in ref. 37 and 38. Conductivities were calculated according to $\sigma=j / F$, where $j$ and $F$ are the current density and electric field, respectively. The Seebeck coefficient was obtained by applying a linear temperature gradient of various magnitudes along the sample and recording the shift of the $j V$-characteristics at the end of a settling time of $\approx 400 \mathrm{~s}$.

\section{Conclusions}

Our findings give a full picture of the interface modification process based on PEDOT:PSS coated with PEIx in large-scale printing. We find that the PEIx can interact with both PSS and PEDOT. The mode of interactions depends on the PSS concentration. We observed that this interface reaction results in a pinning effect to stabilize the surface morphology of PEDOT:PSS, which could be advantageous for suppressing the redistribution of PSS during printing of successive layers. On the other hand, these interactions are found to be the origin of a work function reduction, beside the dipole effect. Although a PEIx layer can cause de-doping, the redoping effect by oxygen is found on the low work function surface, which contributed to the decrease in device performance. This has implications for future materials design and process development for upscaling organic optoelectronic device production.

\section{Conflicts of interest}

There are no conflicts to declare.

\section{Acknowledgements}

The work was funded by the Swedish Energy Agency, the Swedish Science Council, the Swedish Strategic Research Foundation through the project SiOs, the Knut and Alice Wallenberg foundation (KAW) through generous instrument grants, a Wallenberg Scholar grant to O. I., and a Proof of Concept project for upscaling organic solar cells.

\section{References}

1 R. Søndergaard, M. Hösel, D. Angmo, T. T. Larsen-Olsen and F. C. Krebs, Mater. Today, 2012, 15, 36-49.

2 C. Duan, K. Zhang, C. Zhong, F. Huang and Y. Cao, Chem. Soc. Rev., 2013, 42, 9071-9104.

3 G. Yang, H. Tao, P. Qin, W. Ke and G. Fang, J. Mater. Chem. A, 2016, 4, 3970-3990.

4 Y. Zhou, C. Fuentes-Hernandez, J. Shim, J. Meyer, A. J. Giordano, H. Li, P. Winget, T. Papadopoulos, H. Cheun, J. Kim, M. Fenoll, A. Dindar, W. Haske, E. Najafabadi, T. M. Khan, H. Sojoudi, S. Barlow, S. Graham, J. Brédas, S. R. Marder, A. Kahn and B. Kippelen, Science, 2012, 336, 327-332.

5 L. Lucera, P. Kubis, F. W. Fecher, C. Bronnbauer, M. Turbiez, K. Forberich, T. Ameri, H.-J. Egelhaaf and C. J. Brabec, Energy Technol., 2015, 3, 373-384.

6 B. J. Worfolk, S. C. Andrews, S. Park, J. Reinspach, N. Liu, M. F. Toney, S. C. B. Mannsfeld and Z. Bao, Proc. Natl. Acad. Sci. U. S. A., 2015, 112, 14138-14143.

7 J. Hwang, F. Amy and A. Kahn, Org. Electron., 2006, 7, 387-396. 8 A. M. Nardes, M. Kemerink, R. A. J. Janssen, J. A. M. Bastiaansen, N. M. M. Kiggen, B. M. W. Langeveld, A. J. J. M. van Breemen and M. M. de Kok, Adv. Mater., 2007, 19, 1196-1200.

9 J. Ouyang, Displays, 2013, 34, 423-436. 
10 A. Elschner, S. Kirchmeyer, W. Lövenich, U. Merker and K. Reuter, PEDOT: Principles and Applications of an Intrinsically Conductive Polymer, CRC Press, 2010.

11 Z. Tang, A. Elfwing, A. Melianas, J. Bergqvist, Q. Bao and O. Inganäs, J. Mater. Chem. A, 2015, 3, 24289-24296.

12 E. Bundgaard, O. Hagemann, M. Manceau, M. Jørgensen and F. C. Krebs, Macromolecules, 2010, 43, 8115-8120.

13 M. M. Voigt, R. C. I. Mackenzie, C. P. Yau, P. Atienzar, J. Dane, P. E. Keivanidis, D. D. C. Bradley and J. Nelson, Sol. Energy Mater. Sol. Cells, 2011, 95, 731-734.

14 J. E. Lewis, E. Lafalce, P. Toglia and X. Jiang, Sol. Energy Mater. Sol. Cells, 2011, 95, 2816-2822.

15 K. M. Kim, S. Ahn, W. Jang, S. Park, O. O. Park and D. H. Wang, Sol. Energy Mater. Sol. Cells, 2018, 176, 435-440.

16 Y. Wang, W. Lan, N. Li, Z. Lan, Z. Li, J. Jia and F. Zhu, Adv. Eng. Mater., 2019, 9, 1900157.

17 X. Zhu, L. Hu, W. Wang, X. Jiang, L. Hu and Y. Zhou, ACS Appl. Eng. Mater., 2019, 2, 7602-7608.

18 J. Bergqvist, T. Österberg, A. Melianas, L. Ever Aguirre, Z. Tang, W. Cai, Z. Ma, M. Kemerink, D. Gedefaw, M. R. Andersson and O. Inganäs, npj Flexible Electron., 2018, 4, 1-8.

19 L. Ouyang, C. Musumeci, M. J. Jafari, T. Ederth and O. Inganäs, ACS Appl. Mater. Interfaces, 2015, 7, 19764-19773.

20 L. S. C. Pingree, B. A. MacLeod and D. S. Ginger, J. Phys. Chem. C, 2008, 112, 7922-7927.

21 J. Ouyang, Q. Xu, C.-W. Chu, Y. Yang, G. Li and J. Shinar, Polymer, 2004, 45, 8443-8450.

22 S. Fabiano, S. Braun, X. Liu, E. Weverberghs, P. Gerbaux, M. Fahlman, M. Berggren and X. Crispin, Adv. Mater., 2014, 26, 6000-6006.

23 E. Mitraka, M. J. Jafari, M. Vagin, X. Liu, M. Fahlman, T. Ederth, M. Berggren, M. P. Jonsson and X. Crispin, J. Mater. Chem. A, 2017, 5, 4404-4412.
24 P. Demir, S. Onde and F. Severcan, Spectrochim. Acta, Part A, 2015, 135, 757-763.

25 Practical Guide to Chemometrics, ed. P. Gemperline, CRC/ Taylor \& Francis, Boca Raton, 2nd edn, 2006.

26 G. Zuo, X. Liu, M. Fahlman and M. Kemerink, Adv. Funct. Mater., 2018, 28, 1703280.

27 Z. Li, F. Qin, T. Liu, R. Ge, W. Meng, J. Tong, S. Xiong and Y. Zhou, Org. Electron., 2015, 21, 144-148.

28 S. Fabiano, H. Usta, R. Forchheimer, X. Crispin, A. Facchetti and M. Berggren, Adv. Mater., 2014, 26, 7438-7443.

29 M. Lögdlund, R. Lazzaroni, S. Stafström, W. R. Salaneck and J.-L. Brédas, Phys. Rev. Lett., 1989, 63, 1841-1844.

30 J. Huang, P. F. Miller, J. S. Wilson, A. J. de Mello, J. C. de Mello and D. D. C. Bradley, Adv. Funct. Mater., 2005, 15, 290-296.

31 O. Bubnova, Z. U. Khan, A. Malti, S. Braun, M. Fahlman, M. Berggren and X. Crispin, Nat. Mater., 2011, 10, 429-433.

32 B. Winther-Jensen, O. Winther-Jensen, M. Forsyth and D. R. MacFarlane, Science, 2008, 321, 671-674.

33 J. Gustafsson, B. Liedberg and O. Inganas, Solid State Ionics, 1994, 69, 145-152.

34 Y. Xia, X. Xu, L. E. Aguirre and O. Inganäss, J. Mater. Chem. A, 2018, 6, 21186-21192.

35 Y. Galagan, T. M. Eggenhuisen, M. J. J. Coenen, A. F. K. V. Biezemans, W. J. H. Verhees, S. C. Veenstra, W. A. Groen, R. Andriessen and R. A. J. Janssen, J. Mater. Chem. A, 2015, 3, 20567-20578.

36 W. Tress, S. Corvers, K. Leo and M. Riede, Adv. Energy Mater., 2013, 3, 873-880.

37 G. Zuo, O. Andersson, H. Abdalla and M. Kemerink, Appl. Phys. Lett., 2018, 112, 083303.

38 S. van Reenen and M. Kemerink, Org. Electron., 2014, 15, 2250-2255. 